\title{
ELECTRON BEAM DYNAMICS FOR A LOW-ENERGY ELECTRON LINAC
}

\author{
A.F.J. Hammen, J.I.M. Botman, R.W. de Leeuw, H.L. Hagedoorn, W.H.C. Theuws. \\ Eindhoven University of Technology, Cyclotron Laboratory, \\ P.O. Box 513, 5600MB Eindhoven, The Netherlands.
}

\begin{abstract}
Linac characteristics have been calculated from linac structure dimensions, and have been inserted in the power diffusion equation. Assuming a constant beam current along the linac, the EM wave power has been retreived. Numerical computer simulations, using the EM wave power as input, have been used to calculate the beam current along the linac, which has been inserted in the power diffusion equation again. In particular these procedures have been applied to the Eindhoven $10 \mathrm{MeV}$ linac, for which extensive measurements have been carried out. The calculation process converges after a few iterations and yields the load line, and the radial and longitudinal emittances, which are in agreement with the measurements.
\end{abstract}

\section{INTRODUCTION}

At the Eindhoven University of Technology, the Racetrack Microtron Eindhoven (RTME) [1] will be coupled to the Eindhoven $10 \mathrm{MeV}, 2998 \mathrm{MHz}$ travelling-wave linac (type M.E.L. SL75/10). Therefore, linac beam characteristics, in particular the load line, radial and longitudinal emittance have been simulated and measured. In the simulations, space charge effects have been neglected, because of the rather low beam current. Simulations have been compared to calculations based on Hamiltonian theory [2] as well, with an excellent agreement between these methods [3]. Simulations have been carried out with the code Parmela [4].

\section{EM WAVE POWER ALONG THE LINAC}

For Parmela simulations, the amplitude of the longitudinal component of the electric field, $E_{z}$, as a function of the longitudinal co-ordinate, $z$, must be inserted in the input file.

This amplitude is obtained from the power of the EM wave, $P$, in the linac as a function of $z$, which is calculated using the power diffusion equation [5]:

$$
\frac{d P(z)}{d z}=-\frac{\omega_{\phi_{0}}}{v_{g} Q_{010}} P(z)-I_{b}(z) \cos \left(\phi_{a}\right) \sqrt{\frac{\omega_{\phi_{0}}}{v_{g} Q_{010}} r_{s h} P(z)} .
$$

The parameters $\omega_{\phi_{0}}, v_{g}, Q_{010}$ and $r_{s h}$, which stand for the EM wave frequency of the linac operating in the $\phi_{0^{-}}$ mode, the group velocity of the EM wave, the unloaded quality factor of a $\mathrm{TM}_{010}$ wave in a pillbox corresponding to a linac cell and the shunt impedance per unit lenth, are calculated from the linac structure dimensions [6].

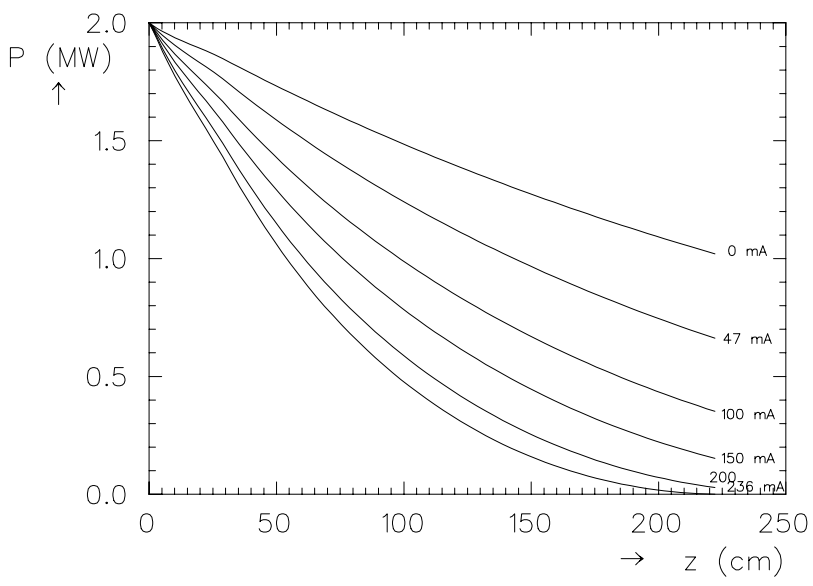

Figure 1: The EM wave power in the Eindhoven $10 \mathrm{MeV}$ linac as a function of longitudinal co-ordinate for various values of final beam current (indicated in the figure). The power at $z=0$ is $2 \mathrm{MW}$, corresponding to the magnetron power.

Assuming a constant beam current $I_{b}(\mathrm{z})$ along the linac and a mean accelerating phase $\phi_{a}=30^{\circ}$ in equation (1), $E_{z}(z)$ is calculated, using [5]:

$$
E_{z}(z)=\sqrt{\frac{\omega_{\phi_{0}}}{v_{g} Q_{010}} r_{s h} P(z)} .
$$

$E_{z}(z)$ is inserted in the Parmela input file yielding a first approximation for $I_{b}(z)$, which is inserted in equation (1), yielding a better approximation for $E_{z}(z)$. This $E_{z}(z)$ is inserted in Parmela again. In this way, $P(z)$ is calculated in an iterative process, which is convergent within $1 \%$ in three steps.

An approximation of the mean energy increase of the electrons, $\Delta E$, is given by:

$$
\Delta E=\int_{z=0}^{L} E_{z}(z) \cos \left(\phi_{a}\right) d z,
$$

in which $L$ is the total length of the linac. It appears that $I_{b}(z)$ does not depend much on $\phi_{a}$, for a fixed final beam current. So $\phi_{a}$ is calculated by matching the mean final energy from Parmela and from equation (3), using $I_{b}(z)$ determined iteratively.

With this value of $\phi_{a}$, which extends from $30^{\circ}$ at $I_{b}(z)=$ 0 to $35^{\circ}$ at the maximum final beam current of $236 \mathrm{~mA}$, one more iteration is applied. The results of the $P(z)$ cal- 
culations for several final beam currents are presented in figure 1 .

\section{LOAD LINE}

The simulated load line is constructed by determining the energy value of the peak in the energy spectrum of Parmela simulations at different final beam currents. The measurement of the load line is performed in a standard spectrometer measurement using a dipole magnet with a slit behind it to select the energy and a Faraday cup, to measure the current. At different beam currents, the energy of the peak has been measured. Results are presented in figure 2 .

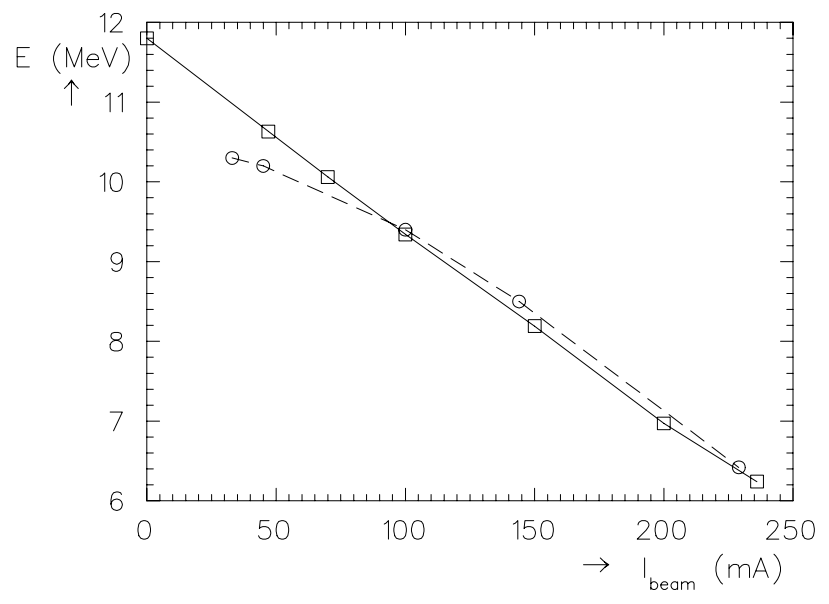

Figure 2: Simulated ( $\square$ ) and measured (o) load lines of the Eindhoven $10 \mathrm{MeV}$ linac.

Above $75 \mathrm{~mA}$, simulations and measurements agree well. Differences at lower beam currents may be due to measurement inaccuracies and to uncertainties in shunt impedance calculations.

\section{RADIAL EMITTANCE}

Using Parmela, the $90 \%$ radial emittance, $\epsilon_{90}$, has been simulated at a $70 \mathrm{~mA}$ (corresponding to $10 \mathrm{MeV}$ electrons) setting and is presented in figure 3 . The emittance yields $\epsilon_{90}=18.5 \mathrm{~mm} \cdot \mathrm{mrad}$. Measurements have been performed, using a set-up consisting of two movable slits in the horizontal and vertical plane at a distance of $58 \mathrm{~cm}$ from each other, with a Faraday cup behind the second slit, to measure the current. The measurements have been corrected for the finite slit thickness and results have been fitted to an ellipse [3]. Results in the transversal $x$ - and $y$-directions are: $\epsilon_{90, x}=11 \mathrm{~mm} \cdot \mathrm{mrad}$ and $\epsilon_{90, y}=17 \mathrm{~mm} \cdot \mathrm{mrad}$.

Differences between simulation and measurements are due to measurement inaccuracy, to the absense of space charge calculations and to the fact that Parmela uses a Floquet series expansion of the electric field, which is not completely valid in the a-periodic initial part of the linac. Differences between $x$ and $y$ measurements are attributed to measuring inaccuracies, to structure imperfections and to

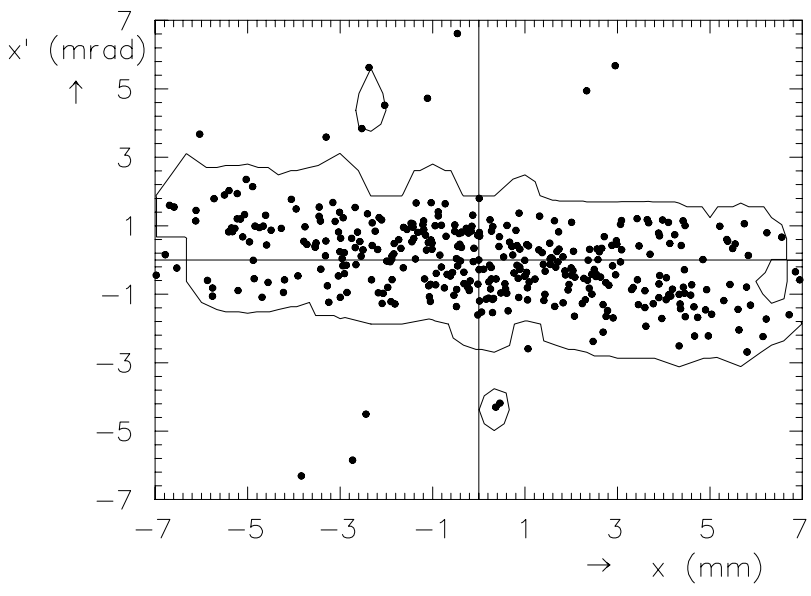

Figure 3: The simulated radial emittance of the Eindhoven $10 \mathrm{MeV}$ linac, the contour determines the $90 \%$ emittance, the dots represent the simulated test particles.

non-rotational symmetric influences, like the electron gun and the coupling in of the RF power.

\section{LONGITUDINAL EMITTANCE}

Using Parmela, the longitudinal emittance has been simulated at a $70 \mathrm{~mA}(10 \mathrm{MeV})$ setting and is presented in figure 4 .

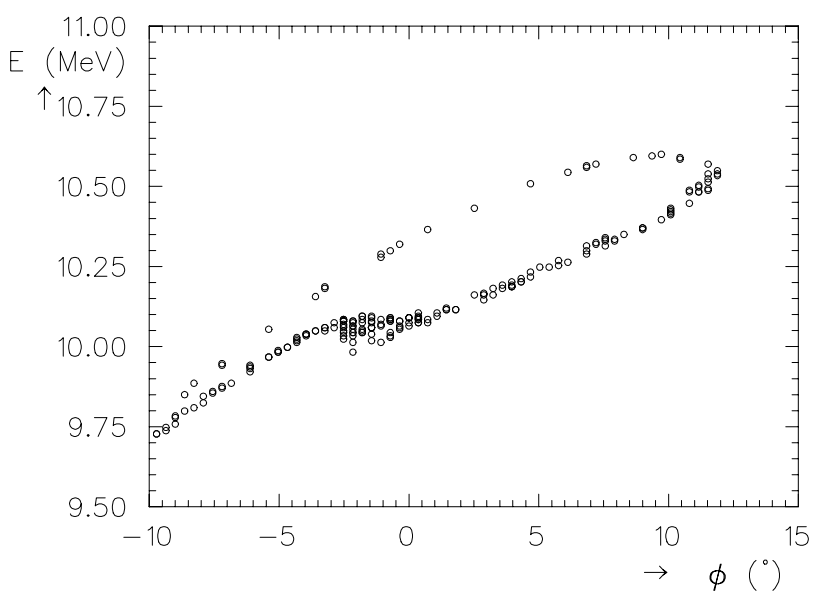

Figure 4: The simulated longitudinal emittance, presented as phase-energy relation, of the Eindhoven $10 \mathrm{MeV}$ linac.

From the longitudinal emittance the energy spectrum can be constructed. This spectrum has also been measured, using the same set-up as in the load line measurement. However, due to the magnitude of the radial emittance, the energy spectrum can not be measured sufficiently accdurately, using this set-up. Therefore, it has been calculated what signal would be measured, assuming the simulated spectrum as input for the measurement set-up [3]. The simulated and the measured energy spectrum as well as the 
calculated measurement spectrum, assuming the simulated spectrum as input, are presented in figure 5.

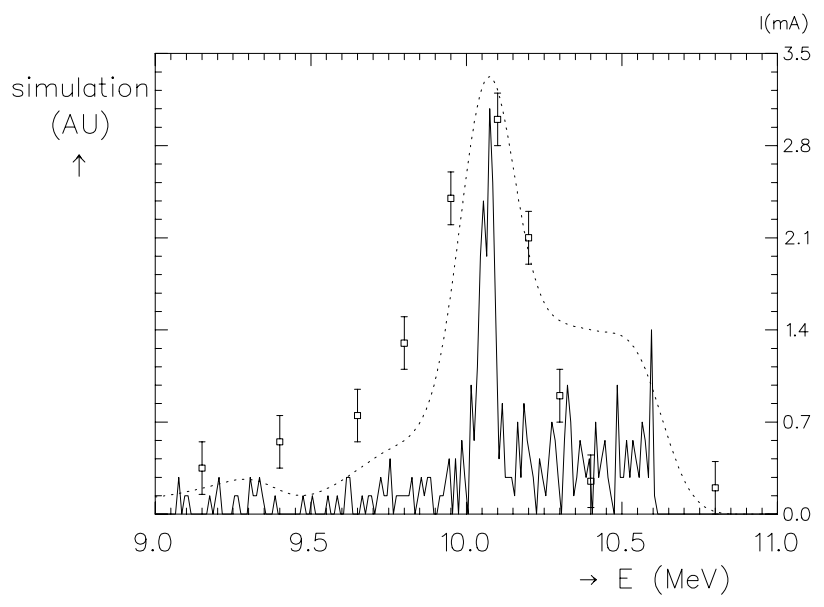

Figure 5: The simulated energy spectrum (solid line), the simulated energy spectrum as would be measured in the used set-up (dotted line) and the measured spectrum ( $\square$ ) (units on right axis) of the Eindhoven $10 \mathrm{MeV}$ linac.

The agreement between measured spectrum and calculated measurement spectrum is rather good, from which it is concluded that the real energy spectrum is about equal to the simulated spectrum. Furthermore, it follows that the calculation of the longitudinal is quite realistic.

\section{INJECTION PHASE DEPENDENCY}

In the initial part of the linac, particles are homogeneously distributed over the RF wave, because of the fact that the RF period is much shorter than the duration of macro pulse fed to the electron gun. Each injection phase interval leads to a specific contribution in the final energy spectrum and the final total emittance. By selecting an optimal $50^{\circ}$ part of the total injection phase distribution, a final beam is obtained with a $3.7^{\circ}$ final phase spread and a $1.3 \%$ final energy spread, the $90 \%$ radial emittance of this beam is $8.1 \mathrm{~mm} \cdot \mathrm{mrad}$. Thus a much higher beam quality is obtained, compared to figures (3) and (4). Injection of this higher quality beam in the RTME is prefered, because of better matching and a higher quality beam at the end of the RTME.

\section{CONCLUSIONS}

Linac beam characteristics have been determined, with measurements and simulations. There is a good agreement between these methods, which means that the method used to calculate the EM wave power along the linac is correct and that the simulated linac characteristics are quite reliable.

Comparing the beam characteristics to the acceptance of the RTME [7], the current that will be accepted in RTME is more than adequate [3] and [8].

\section{REFERENCES}

[1] Theuws W.H.C., Botman J.I.M., Hagedoorn H.L., Leeuw R.W. de, Timmermans C.J., The $75 \mathrm{MeV}$ racetrack microtron Eindhoven, LINAC 96. Proc. of the XVIII International Linear Accelerator Conference, Geneva (1996), 92-94.

[2] Hagedoorn H.L., Botman J.I.M., Leeuw R.W. de, Stas R.J.W., Orbit dynamics in a low energy electron linear accelerator, EPAC 96. Proc. of the fifth European Particle Accelerator Conference, Barcelona (1996), 944-946.

[3] Hammen A.F.J., Particle dynamics in the $10 \mathrm{MeV}$ TUE electron linac, Internal Report, M.Sc. Thesis, Eindhoven University of Technology (1997).

[4] Parmela, Reference manual, Los Alamos Accelerator Code Group, Los Alamos National Laboratory, Los Alamos, USA (1987).

[5] Leiss J.E., Beam loading and transient behavior in travelling wave electron linear accelerators in Linear accelerators, Lapostolle P.M., Septier A.L. eds., North Holland Publ. Comp., Amsterdam (1970) 147-172.

[6] Gao J., Analytical formulae for the resonant frequency changes due to opening apertures on cavity walls, EPAC 92. Proc. of the third European Particle Accelerator Conference, Berlin (1992) 865-867.

[7] Webers G.A., Design of an electron-optical system for a $75 \mathrm{MeV}$ racetrack microtron, Ph.D. Thesis Eindhoven University of Technology (1994).

[8] Theuws W.H.C., Kemper A.H., Ridderhof E.J., Timmermans C.J., Electronic detection circuit for a stripline beam position monitor, EPAC 96. Proc. of the fifth European Particle Accelerator Conference, Barcelona (1996), 1618-1620. 